\title{
Cell Association in Small Heterogeneous Networks: Downlink Sum Rate and Min Rate Maximization
}

\author{
Steven Corroy*, Laetitia Falconetti** and Rudolf Mathar* \\ *Institute for Theoretical Information Technology, RWTH Aachen University, Aachen, Germany \\ **Ericsson Research, Aachen, Germany \\ \{corroy, mathar\}@ti.rwth-aachen.de, laetitia.falconetti@ericsson.com
}

\begin{abstract}
This paper considers the problem of associating users, in an heterogeneous network, to either a macro node or a pico node within a tightly coordinated cell cluster. We introduce a new theoretical framework to model this problem for the downlink and derive upper bounds for achievable sum rate and minimum rate using convex optimization. Further we propose heuristics, consisting in dynamic cell association, enabling to achieve performance close to the upper bounds. Finally we implement these heuristics in an LTE simulator and show the potential of such dynamic cell association for a small LTE network.
\end{abstract}

\section{INTRODUCTION}

Heterogeneous Network (HetNet) is seen as a promising way to meet the increasing demand for mobile broadband traffic in cellular networks. In HetNet, a typical setup consists of a network with a macro node complemented with pico nodes. The goal of the pico nodes is to provide higher capacity in areas with high user equipment (UE) density. The considered pico nodes use the same frequency band as the macro node, but have a much smaller transmit (Tx) power.

In such a HetNet, a fundamental problem is the one of associating UEs, either with the macro node or with a pico node. In 3GPP Long Term Evolution (LTE) networks, a UE is associated with the node whose signal is received with the largest average strength. We call this algorithm the best $S N R$ heuristic. It ensures robustness of the user transmission towards interference. However, due to the Tx power imbalance between macro and pico nodes, this cell association only allows a small fraction of users to connect to the pico nodes [1]. In other words, it leads to a load imbalance between the macro and pico nodes, which limits the user throughput.

A modified cell association has been evaluated within 3GPP [1], [2]. It increases the coverage area of pico nodes by adding a fixed bias to the measured received $(\mathrm{Rx})$ power of pico nodes. Thus, a larger fraction of users is served by low power nodes, and a better load balancing is achieved between the macro and pico nodes. However, with this static modification to the cell association, certain users that are connected with the pico nodes, receive stronger signals from the macro node and the achieved better load balancing may not compensate a lower Signal to Interference and Noise Ratio (SINR) resulting from the increased interference level.

The goal of the present work is to determine a dynamic scheme for cell association for the downlink in HetNets,

This work was partly supported by the UMIC research cluster of the RWTH Aachen University. which optimizes the system performance (in terms of sum rate and min rate) and to analyze the trade-off between the load balancing and the interference level.

Cell association has been studied for load balancing in $3 \mathrm{G}$ networks. In [3] and [4], the authors address a setup in which, the base stations (BSs) serve a single UE at each time slot and derive a centralized and distributed algorithm respectively. In [5] and [6], the authors assume a proportional fair scheduler at each BS and derive optimal cell association by solving a sequence of Boolean linear programs.

The main difference between the present work and [3][6], is that they do not consider a HetNet scenario, where there is a large power imbalance between nodes and a specific structure of the interference. Another important point is that we do not directly optimize the load balancing, but rather we want to understand the influence of the trade-off between load balancing and interference on other metrics like, e.g, the sum rate. In contrast to [3], [4] we address the impact of having more or less users served at the same time on the same resource by a specific node. Finally as opposed to [5], [6], we bound the system performance using convex optimization and achieve this bound using a heuristic with a complexity linear in the number of users.

The contribution of the present paper is manifolds. We propose a new theoretical framework to analyze dynamic cell association in HetNet and derive upper bounds on achievable sum rate and min rate using convex optimization. We describe low complexity heuristics, which achieve performance close to the upper bounds. We verify our results through numerical evaluations and analyze the offloading potential of increasing the pico coverage area and the associated rise of interference. Finally we implement these heuristics in an LTE simulator to show the potential of dynamic cell association in a real setup.

Section II and III describe our setup and optimization problems. Section IV and V derive upper bounds on the solution of these problems, as well as heuristics approaching these bounds. Section VI presents numerical results and Section VII concludes this work.

\section{DYNAMIC CELL ASSOCIATION WITHIN A TIGHTLY COORDINATED CELL CLUSTER}

Since the goal of this work is to understand the impact of cell association and load balancing in HetNet, with respect to UE bitrate metrics, we consider a simple network composed of a macro node and one pico node. This is an important first 


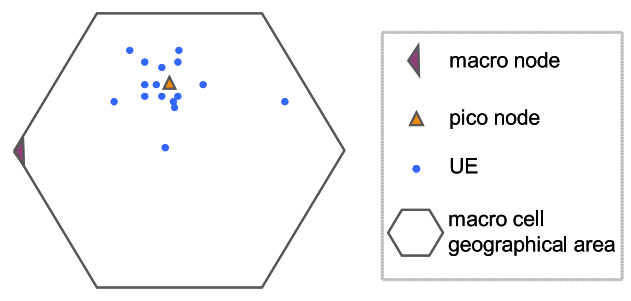

Fig. 1. Scenario with tightly coordinated macro and pico nodes.

step toward analyzing larger networks. Figure 1 illustrates the considered scenario.

The dynamic cell association takes its decision by considering the long-term signal measurements and the total number of users within the network. Therefore, it is not expected to be performed very often, but only when the number of active UEs changes (UE with new transmission or with finished transmission) or when the long-term signal measurements have significantly changed.

On the contrary, the resource scheduling is performed at each node on a much more frequent basis. In order to decouple the association problem and the scheduling problem, we first assume that the macro and pico nodes share their bandwidth equally among all associated UEs, which corresponds to a Round Robin scheduler. This enables us to upper bound the achievable sum rate and min rate of the network. We then implement our dynamic cell association on an LTE simulator and verify that this decoupling is a meaningful assumption. Note that this approach extends easily to another scheduler, e.g., proportional fairness, by simply scaling the UE rates with a weight proportional to their past rate.

Dynamic cell association requires a tight coordination between nodes to exchange the required information and react quickly to a changed situation. It may also be preferable that the different nodes appear to the user as the same cell, as described in [7]. This makes the handover invisible to the user and enables to shorten the handover delay and avoid the usual handover signaling overhead. In that case the cell association is actually a point selection since, for the UEs, there is only one cell. In this paper, we assume that such tight coordination is possible between the macro node and the pico node.

\section{SYSTEM MODEL AND OPTIMIZATION PROBLEM}

We consider a cooperation cluster with one macro node (in the following node 1 ) with a transmit power $\sigma_{1}$ and one pico node (node 2) with a transmit power $\sigma_{2}<\sigma_{1}$. Each node operates with a bandwidth $B$ at the same frequency band. We assume that the network contains $n$ UEs for which a cell association should be defined.

Consider $\mathbf{x}_{1}, \mathbf{x}_{2} \in\{0,1\}^{n}$ representing the association of the UEs to one or the other node as follows.

$$
\begin{array}{ll}
x_{j, i}=1 & \text { if UE } i \text { is associated with node } j, \\
x_{j, i}=0 & \text { Otherwise. }
\end{array}
$$

A UE $i$ is associated with a single node, therefore $\mathbf{x}_{1}+\mathbf{x}_{2}=\mathbf{1}$, where 1 is a vector of size $n$ containing only ' 1 's.

The goal of the present work is to maximize a function $f(\mathbf{x})$, where $\mathbf{x} \triangleq\left[\mathbf{x}_{1}^{\mathrm{T}} \mathbf{x}_{2}^{\mathrm{T}}\right]^{\mathrm{T}}$. We consider two optimization problems.
In the first one, $f(\mathbf{x})$ represents the sum rate of all users, while in the second problem $f(\mathbf{x})$ represents the minimum user rate.

To get an estimate of the achievable user rate per $\mathrm{Hz}$ with a certain cell association, the equation $\mathbf{C}(\gamma)=\log _{2}(1+\gamma)$ is used. $\gamma$ denotes the user SINR calculated based on the average channel gain, since the cell association mechanism is supposed to be valid over a long term.

If $g_{i j}$ represents the average channel gain between node $j$ and UE $i$, the average received power from the node $j$ at UE $i$ can be expressed as $\hat{\sigma}_{i j}=\sigma_{j} g_{i j}$. Further we define $\sigma_{n}$ as the noise and interference power measured over $B$ at the receiver, where the interference component of $\sigma_{n}$ is an estimate of the interference power received from all cells outside the coordination cluster.

We assume that there is no restriction in the frequency resources that a node can allocate to a user. Consequently, a node always allocates all available frequency resources to its associated UEs at a given time. In other words, as soon as there is one active UE associated with a node, this node creates interference over the whole bandwidth. Accordingly two cases can be observed.

1) All UEs are associated with the same node. In that case, each UE $i$ has a SINR with the associated node $j$ corresponding to $\hat{\sigma}_{i j} / \sigma_{n}$ and an achievable rate per $\mathrm{Hz}$ of $\mathrm{C}\left(\hat{\sigma}_{i j} / \sigma_{n}\right)$.

2) At least one UE is associated with each node. Here, each UE $i$ has a SINR with the associated node $j$ corresponding to $\hat{\sigma}_{i j} /\left(\hat{\sigma}_{i k}+\sigma_{n}\right)$ with $k \neq j$. The achievable rate per $\mathrm{Hz}$ is $\mathrm{C}\left(\hat{\sigma}_{i j} /\left(\hat{\sigma}_{i k}+\sigma_{n}\right)\right)$.

Let the vector $\hat{\mathbf{r}}_{j}=\left(\hat{r}_{j, i}\right)_{1 \leq i \leq n} \in \mathbb{R}^{n}$ contains the achievable downlink rate of all UEs, as if they are scheduled alone with node $j \in\{1,2\}$ and there exists at least one other UE scheduled with the other node.

$$
\hat{r}_{j, i}=B \cdot \mathrm{C}\left(\hat{\sigma}_{i j} /\left(\hat{\sigma}_{i k}+\sigma_{n}\right)\right), j \neq k .
$$

The vector $\tilde{\mathbf{r}}_{j}=\left(\tilde{r}_{j, i}\right)_{1 \leq i \leq n} \in \mathbb{R}^{n}$ contains the achievable downlink rate of all UEs, if they are scheduled alone with node $j$ and no other UE is scheduled with the other node.

$$
\tilde{r}_{j, i}=B \cdot \mathrm{C}\left(\hat{\sigma}_{i j} / \sigma_{n}\right), j \neq k .
$$

Finally we define $\mathbf{r}_{1} \triangleq\left[\begin{array}{ll}\hat{\mathbf{r}}_{1}^{\mathrm{T}} & \mathbf{0}^{\mathrm{T}}\end{array}\right]^{\mathrm{T}}, \quad \mathbf{r}_{2} \triangleq\left[\begin{array}{ll}\mathbf{0}^{\mathrm{T}} & \hat{\mathbf{r}}_{2}^{\mathrm{T}}\end{array}\right]^{\mathrm{T}}$, $\mathbf{1}_{1} \triangleq\left[\begin{array}{ll}\mathbf{1}^{\mathrm{T}} & \mathbf{0}^{\mathrm{T}}\end{array}\right]^{\mathrm{T}}$ and $\mathbf{1}_{2} \triangleq\left[\begin{array}{ll}\mathbf{0}^{\mathrm{T}} & \mathbf{1}^{\mathrm{T}}\end{array}\right]^{\mathrm{T}}$, where $\mathbf{0}$ is a vector of size $n$ containing only ' 0 's. .

\section{A. Sum rate maximization}

Using the previously defined variables we can formulate the problem of finding the cell association, which maximizes the sum rate of all UEs as follows. First solve the problem

$$
\begin{array}{cl}
\underset{\mathbf{x}}{\operatorname{maximize}} & \frac{\mathbf{r}_{1}^{\mathrm{T}} \mathbf{x}}{\mathbf{1}_{1}^{\mathrm{T}} \mathbf{x}}+\frac{\mathbf{r}_{2}^{\mathrm{T}} \mathbf{x}}{\mathbf{1}_{2}^{\mathrm{T}} \mathbf{x}} \\
\text { subject to } & {[\mathbf{I} \mathbf{I}] \mathbf{x}=\mathbf{1}, \mathbf{1}_{1}^{\mathrm{T}} \mathbf{x} \geq 1, \mathbf{1}_{2}^{\mathrm{T}} \mathbf{x} \geq 1} \\
& x_{i} \in\{0,1\}, i=1, \ldots, 2 n,
\end{array}
$$

where $\mathbf{I}$ is the identity matrix of size $n$. 
The division by $\mathbf{1}_{j}^{\mathrm{T}} \mathbf{x}$ in Equation (4) reflects the fact that the time-frequency resources of a node have to be shared among the users associated to this node. Consequently the achievable bitrate for a user decreases when more users are associated with the same node. In Equation (4) an equal share of the node resources among the served users is assumed. This corresponds to the well-known Round Robin scheduler.

Let us call $p^{\star}$ the optimal value of Equation (4) and $\mathrm{x}^{\star}$ the corresponding optimal cell association. We then compute $\max \left\{p^{\star}, \frac{\mathbf{1}^{\mathrm{T}} \tilde{\mathbf{r}}_{1}}{n}, \frac{\mathbf{1}^{\top} \tilde{\mathbf{r}}_{2}}{n}\right\}$. If $p^{\star}$ is the maximum, the optimal cell association is given by $\mathbf{x}^{\star}$. If $\frac{\mathbf{1}^{\mathrm{T}} \tilde{\mathbf{r}}_{j}}{n}$ is the maximum, the optimal cell association is when all UEs are with node $j$.

\section{B. Minimum rate maximization}

Similarly we can formulate the problem of finding the cell association, which maximizes the minimum rate among all UEs as follows. First find $p^{\star}$, the optimal value of the problem

$$
\begin{array}{cl}
\underset{\mathbf{x}}{\operatorname{maximize}} & \min _{i}\left(\frac{r_{1, i} x_{i}}{\mathbf{1}_{1}^{\mathrm{T}} \mathbf{x}}+\frac{r_{2, i+n} x_{i+n}}{\mathbf{1}_{2}^{\mathrm{T}} \mathbf{x}}\right) \\
\text { subject to } & {[\mathbf{I} \mathbf{I}] \mathbf{x}=\mathbf{1}, \mathbf{1}_{1}^{\mathrm{T}} \mathbf{x} \geq 1, \mathbf{1}_{2}^{\mathrm{T}} \mathbf{x} \geq 1} \\
& x_{i} \in\{0,1\}, i=1, \ldots, 2 n .
\end{array}
$$

Then compute $\max \left\{p^{\star}, \min _{i}\left(\frac{\tilde{r}_{1, i}}{n}\right), \min _{i}\left(\frac{\tilde{r}_{1, i}}{n}\right)\right\}$ to find the optimal cell association.

Problems (4) and (5) are nonconvex and therefore hard to solve exactly.

\section{Cell association for sum Rate maximization}

We derive an upper bound on the solution of problem (4) and develop a heuristic approaching this upper bound.

\section{A. Upper bound}

First we rewrite problem (4) as follows.

$$
\begin{array}{ll}
\underset{\mathbf{x}}{\operatorname{maximize}} & \frac{\mathbf{r}_{1}^{\mathrm{T}} \mathbf{x x}^{\mathrm{T}} \mathbf{1}_{2}+\mathbf{r}_{2}^{\mathrm{T}} \mathbf{x} \mathbf{x}^{\mathrm{T}} \mathbf{1}_{1}}{\mathbf{1}_{1}^{\mathrm{T}} \mathbf{x x}^{\mathrm{T}} \mathbf{1}_{2}} \\
\text { subject to } & {[\mathbf{I} \mathbf{I}] \mathbf{x}=\mathbf{1}, \mathbf{1}_{1}^{\mathrm{T}} \mathbf{x} \geq 1, \mathbf{1}_{2}^{\mathrm{T}} \mathbf{x} \geq 1} \\
& x_{i} \in\{0,1\}, i=1, \ldots, 2 n .
\end{array}
$$

We then proceed to a variable change $\mathbf{X} \triangleq \mathbf{x x}^{\mathrm{T}}$. Furthermore, since the constraint $\mathbf{X}=\mathrm{xx}^{\mathrm{T}}$ is equivalent to the two constraints $\mathbf{X} \succcurlyeq \mathbf{0}$ and $\operatorname{rank}(\mathbf{X})=1$, we replace these constraints and get the following nonconvex optimization problem.

$$
\begin{array}{cl}
\underset{\mathbf{X}}{\operatorname{maximize}} & \frac{\mathbf{r}_{1}^{\mathrm{T}} \mathbf{X} \mathbf{1}_{2}+\mathbf{r}_{2}^{\mathrm{T}} \mathbf{X} \mathbf{1}_{1}}{\mathbf{1}_{1}^{\mathrm{T}} \mathbf{X} \mathbf{1}_{2}} \\
\text { subject to } & {[\mathbf{I} \mathbf{I}] \operatorname{diag}(\mathbf{X})=\mathbf{1}} \\
& \mathbf{1}_{1}^{\mathrm{T}} \operatorname{diag}(\mathbf{X}) \geq 1, \mathbf{1}_{2}^{\mathrm{T}} \operatorname{diag}(\mathbf{X}) \geq 1 \\
& X_{i j} \in\{0,1\}, i, j=1, \ldots, 2 n \\
& \mathbf{X} \succcurlyeq \mathbf{0}, \operatorname{rank}(\mathbf{X})=1 .
\end{array}
$$

As such we cannot solve problem (7). In order to get an upper bound on its solution we do the following.

1) We relax the nonconvex constraints by changing $X_{i j}=\{0,1\}$ to $0 \leq X_{i j} \leq 1$ and dropping the rank constraint $\operatorname{rank}(\mathbf{X})=1$.

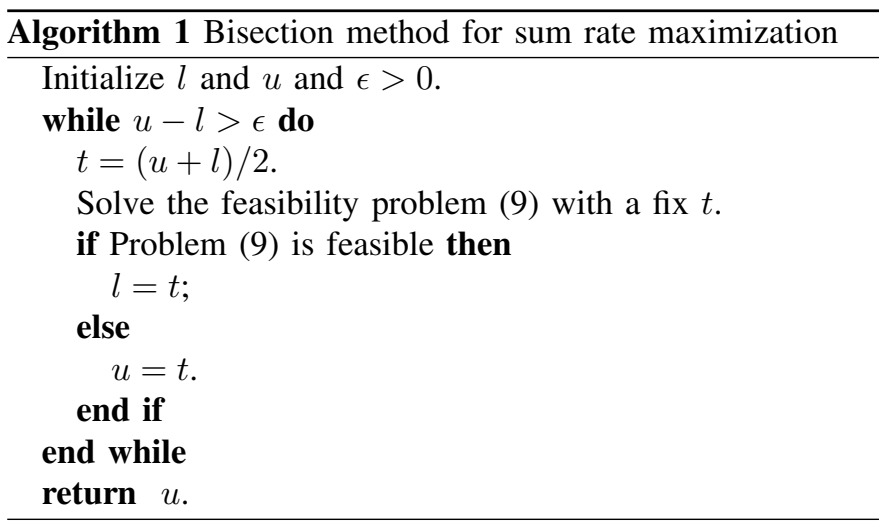

2) We explicitly rewrite constraints on the structure of the problem which disappeared by dropping the rank constraint. Namely we know that $\sum_{i=1}^{2 n} \sum_{j=1}^{2 n} X_{i j}=n^{2}$ since $\mathbf{x}$ had exactly $n$ entries equal to 1 and the other equal to 0 . Furthermore we know that we have at least $n-1$ entries equal to 1 in the upper right corner of $\mathbf{X}$ (equal to $\mathbf{x}_{1} \mathbf{x}_{2}{ }^{\mathrm{T}}$ ) since at least one UE is associated to node 1 or 2 .

We relax problem (7) as follows.

$$
\begin{array}{ll}
\underset{\mathbf{X}}{\operatorname{maximize}} & \frac{\mathbf{r}_{1}^{\mathrm{T}} \mathbf{X} \mathbf{1}_{2}+\mathbf{r}_{2}^{\mathrm{T}} \mathbf{X} \mathbf{1}_{1}}{\mathbf{1}_{1}^{\mathrm{T}} \mathbf{X} \mathbf{1}_{2}} \\
\text { subject to } & {[\mathbf{I} \mathbf{I}] \operatorname{diag}(\mathbf{X})=\mathbf{1}} \\
& \mathbf{1}_{1}^{\mathrm{T}} \operatorname{diag}(\mathbf{X}) \geq 1, \mathbf{1}_{2}^{\mathrm{T}} \operatorname{diag}(\mathbf{X}) \geq 1 \\
& 0 \leq X_{i j} \leq 1 i, j=1, \ldots, 2 n, \mathbf{X} \succcurlyeq \mathbf{0} \\
& \sum_{i=1}^{2 n} \sum_{j=1}^{2 n} X_{i j}=n^{2}, \sum_{i=1}^{n} \sum_{j=n+1}^{2 n} X_{i j} \geq n-1 .
\end{array}
$$

The problem (8) is quasiconcave [8] since all its constraints are convex and its objective function is quasiconcave. To see this, observe that all superlevel sets of the objective function,

$$
\mathcal{S}_{t}=\left\{\mathbf{X} \mid \mathbf{1}_{1}^{\mathrm{T}} \mathbf{X} \mathbf{1}_{2}>0, \mathbf{r}_{1}^{\mathrm{T}} \mathbf{X} \mathbf{1}_{2}+\mathbf{r}_{2}^{\mathrm{T}} \mathbf{X} \mathbf{1}_{1} \geq t \mathbf{1}_{1}^{\mathrm{T}} \mathbf{X} \mathbf{1}_{2}\right\},
$$

are convex. We can write the following feasibility problem,

find $\mathbf{X}$

$$
\begin{array}{ll}
\text { subject to } & \mathbf{r}_{1}^{\mathrm{T}} \mathbf{X} \mathbf{1}_{2}+\mathbf{r}_{2}^{\mathrm{T}} \mathbf{X} \mathbf{1}_{1} \geq t \mathbf{1}_{1}^{\mathrm{T}} \mathbf{X} \mathbf{1}_{2} \\
& {[\mathbf{I} \mathbf{I}] \operatorname{diag}(\mathbf{X})=\mathbf{1}} \\
& \mathbf{1}_{1}^{\mathrm{T}} \operatorname{diag}(\mathbf{X}) \geq 1, \mathbf{1}_{2}^{\mathrm{T}} \operatorname{diag}(\mathbf{X}) \geq 1 \\
& 0 \leq X_{i j} \leq 1 i, j=1, \ldots, 2 n, \mathbf{X} \succcurlyeq \mathbf{0} \\
& \sum_{i=1}^{2 n} \sum_{j=1}^{2 n} X_{i j}=n^{2}, \sum_{i=1}^{n} \sum_{j=n+1}^{2 n} X_{i j} \geq n-1,
\end{array}
$$

and solve problem (8) efficiently using the bisection method described in Algorithm 1. The final value of $u$ is an upper bound on the optimal value of problem (4).

\section{B. Dynamic range heuristic}

In this heuristic, the users are first sorted according to the difference in the power they received from the nodes of the coordination cluster: $\Delta \hat{\sigma}_{i}=\hat{\sigma}_{i 2}-\hat{\sigma}_{i 1}$. We then compute the 
sum rate achieved by $n+1$ different cell associations, starting with the initial state where all users are associated with node 1. We associate the UE with the largest $\Delta \hat{\sigma}_{i}$ with node 2 , compute the resulting sum rate and repeat this process until all UEs are with node 2 . We select the cell association providing the largest sum rate. This heuristic has a complexity linear in the number of UEs. Interestingly, numerical evaluations in Section VI suggest that it is close to optimal.

\section{Cell association for min. RATE MAXIMIZATION}

\section{A. Upper bound}

Similar to the sum rate maximization problem we can reformulate problem (5) as follows.

$$
\begin{array}{cl}
\underset{\mathbf{X}}{\operatorname{maximize}} & \min _{i}\left(\frac{r_{1, i} \mathbf{X}_{i}^{\mathrm{T}} \mathbf{1}_{2}+r_{2, i+n} \mathbf{X}_{i+n}^{\mathrm{T}} \mathbf{1}_{1}}{\mathbf{1}_{1}^{\mathrm{T}} \mathbf{X} \mathbf{1}_{2}}\right) \\
\text { subject to } & {[\mathbf{I} \mathbf{I}] \operatorname{diag}(\mathbf{X})=\mathbf{1}} \\
& \mathbf{1}_{1}^{\mathrm{T}} \operatorname{diag}(\mathbf{X}) \geq 1, \mathbf{1}_{2}^{\mathrm{T}} \operatorname{diag}(\mathbf{X}) \geq 1 \\
& X_{i j} \in\{0,1\}, i, j=1, \ldots, 2 n \\
& \mathbf{X} \succcurlyeq \mathbf{0}, \operatorname{rank}(\mathbf{X})=1 .
\end{array}
$$

Where $\mathbf{X}_{i}$ is the $i$-th column of $\mathbf{X}$. This problem can be relaxed in the same manner.

$$
\begin{array}{ll}
\underset{\mathbf{X}}{\operatorname{maximize}} & \min _{i}\left(\frac{r_{1, i} \mathbf{X}_{i}^{\mathrm{T}} \mathbf{1}_{2}+r_{2, i+n} \mathbf{X}_{i+n}^{\mathrm{T}} \mathbf{1}_{1}}{\mathbf{1}_{1}^{\mathrm{T}} \mathbf{X} \mathbf{1}_{2}}\right) \\
\text { subject to } & {[\mathbf{I} \mathbf{I}] \operatorname{diag}(\mathbf{X})=\mathbf{1}} \\
& \mathbf{1}_{1}^{\mathrm{T}} \operatorname{diag}(\mathbf{X}) \geq 1, \mathbf{1}_{2}^{\mathrm{T}} \operatorname{diag}(\mathbf{X}) \geq 1 \\
& X_{i j} \in\{0,1\}, i, j=1, \ldots, 2 n, \mathbf{X} \succcurlyeq \mathbf{0} \\
& \sum_{i=1}^{2 n} \sum_{j=1}^{2 n} X_{i j}=n^{2}, \sum_{i=1}^{n} \sum_{j=n+1}^{2 n} X_{i j} \geq n-1 .
\end{array}
$$

Since the term in the min function is quasiconcave and that the minimum of quasiconcave functions is also quasiconcave [8], we can solve problem (11) using the bisection method.

\section{B. Dynamic range heuristic}

The heuristic is close to the one described in section IV-B. For all considered $n+1$ cell associations, the minimum user rate is calculated. The finally selected cell association is the one providing the largest minimum rate.

\section{NUMERICAL RESUlts}

In this section, the dynamic range heuristic for the case of sum rate and min rate maximization is compared with the best SNR heuristic, in which a user $i$ is associated with the node $j$ with the largest average received signal power $\hat{\sigma}_{i j}$. The different cell associations are evaluated in a simplified radio network composed of one macro node with a Tx power of $40 \mathrm{~W}$ and one pico node with a Tx power of $1 \mathrm{~W}$. Each node operates with a $5 \mathrm{MHz}$ bandwidth. The pico node is randomly dropped within the macro cell area with a radius of $167 \mathrm{~m}$.

As proposed in [9], we calculate the average channel gain between the macro node and a user $i$ as $g_{i 1}=-(128.1+$ $\left.37.6 \log \left(d_{i 1}\right)\right)$, while the average channel gain between the

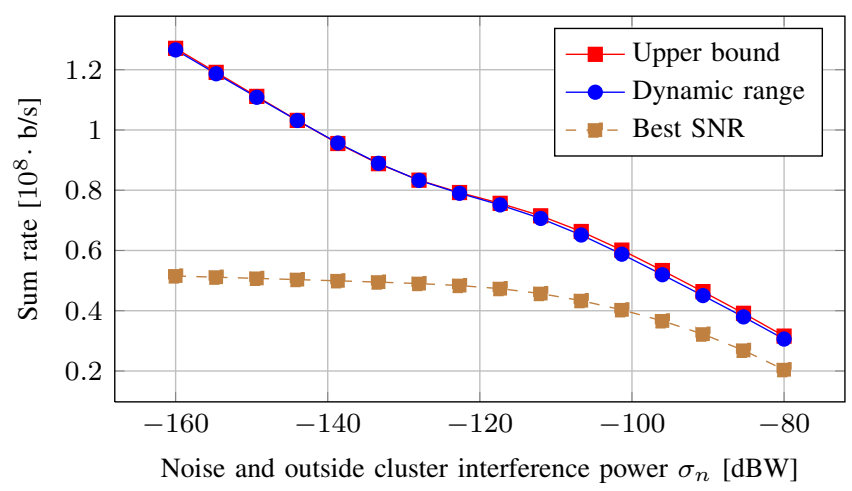

Fig. 2. Sum rate maximization: UE sum rate for different cell associations.

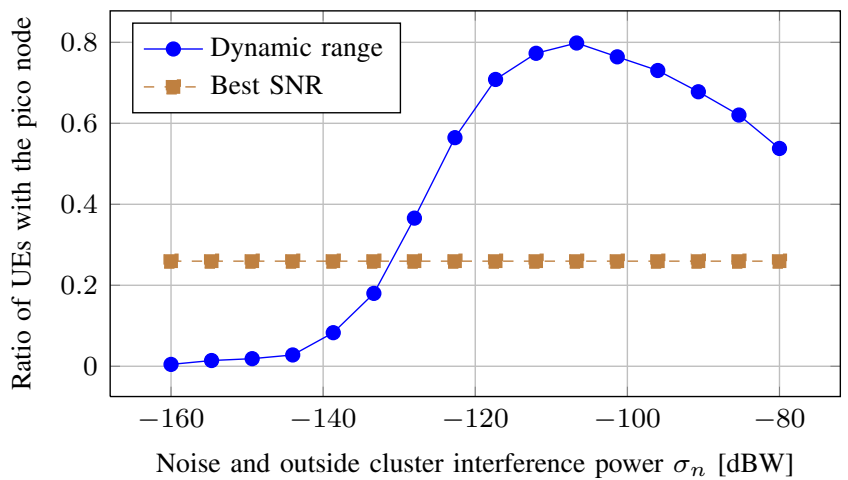

Fig. 3. Sum rate maximization: ratio of UEs with the pico node.

pico node and a user $i$ is given by $g_{i 2}=-(140.7+$ $\left.36.7 \log \left(d_{i 2}\right)\right)$, where $d_{i j}$ denotes the distance between the user $i$ and the node $j$ in $\mathrm{km}$. The users are dropped in a hotspot of $40 \mathrm{~m}$ radius around the pico node with a probability of $2 / 3$.

To produce Figures 2, 3, 4 and 5, $\sigma_{n}$ has been varied from $-160 \mathrm{dBW}$ to $-80 \mathrm{dBW}$. For each value of $\sigma_{n}, 200$ drops of 10 UEs have been performed. For each drop, the corresponding achievable rate for each user with different cell associations has been measured.

Figure 2 shows the average UE sum rate over all drops at different interference and noise levels. The two main points to see from this curve are 1) the dynamic range heuristic outperforms the standard best SNR heuristic and 2) the dynamic range heuristic is actually overlapping the upper bound, such that we can say that this heuristic is nearly optimal.

Figure 3 further illustrates the ratio of pico users depending on the noise and outside cluster interference level. It is observed that the best SNR heuristic provides a pico user ratio of about $26 \%$, although the users have a larger probability $(2 / 3)$ to be in the direct vicinity of the pico node. This is due to the Tx power imbalance between the macro and pico nodes and the proposed average channel gain model of [9]. By contrast, the ratio of pico users changes with $\sigma_{n}$ for the dynamic range association. At low outside cluster interference level, the load is particularly imbalanced since all users are assigned to the macro node. This is to avoid an interference rise, as explained later. At medium interference level, most of the users are served by the pico node. The basic strategy of 


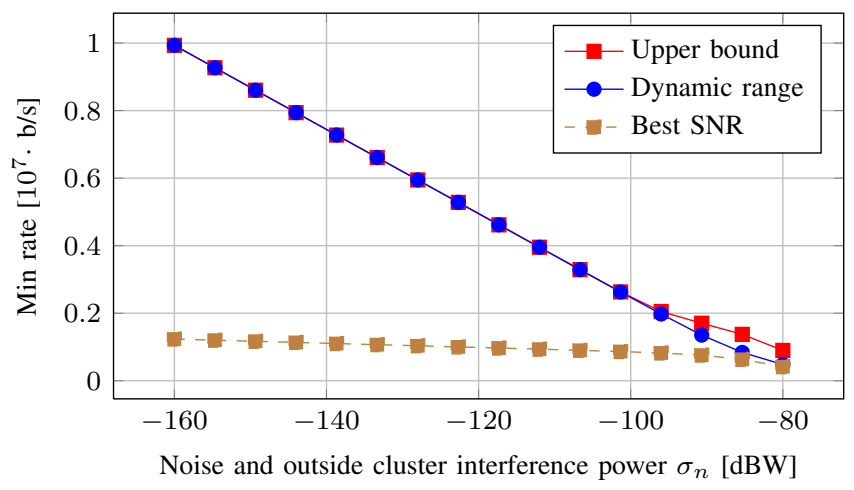

Fig. 4. Min rate maximization: UE min rate for different cell associations.

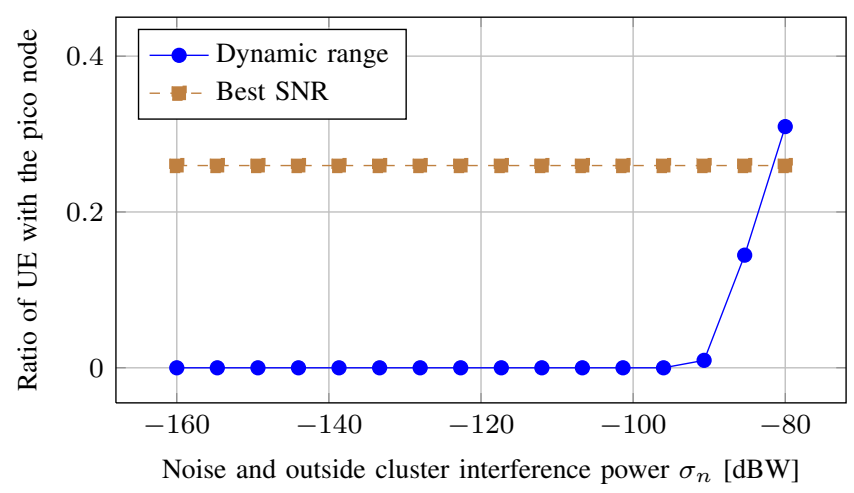

Fig. 5. Min rate maximization: average ratio of UE with the pico node.

the sum rate optimization here is to assign to the macro node only the closest users, so as to achieve large peak user rate for them and boost the global sum rate. At high interference regime, the load is evenly spread among the nodes, since they have a similar share of users.

Figure 4 and 5 show similar results but for the minimum rate optimization problem. There also, the dynamic range heuristic provides a minimum user rate close to the upper bound, while it outperforms the minimum user rate of the best SNR cell association. With the minimum rate optimization, it can be observed that the load is very imbalanced at low and moderate outside cluster interference level. Indeed, this strategy assigns no user to the pico node so as to avoid creating additional intracluster interference by the pico node. In this case, even remote users have a good SINR and therefore a high achievable rate. At high $\sigma_{n}$ however, the load is better shared among nodes. In that case the increased interference caused by the pico node does not affect the SINR so much anymore and more users get assigned to the pico node.

Finally, we simulate a small LTE network composed of one macro node, one pico node and 10 UEs, dropped as previously. Figure 6 shows the cumulative distribution of the achieved user throughput obtained through simulations for the best SNR heuristic and the dynamic range heuristic, both for sum rate and min rate maximization. The same average channel gain equation was used, while the fast fading followed the SCME models defined in [10]. The received noise + outside cluster interference level was set to $-153 \mathrm{dBW}$.

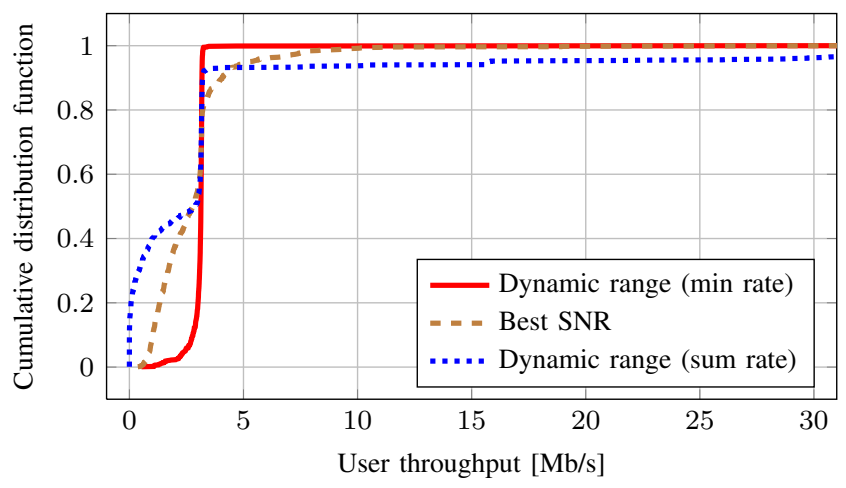

Fig. 6. CDF of UE rate in an LTE network for different cell associations.

We can observe that the sum rate maximization provides good performance for a few macro users at the cost of the other users, while the min rate maximization boosts the rate of cell UEs, yielding a similar user throughput for all users. The cell association with sum rate maximization provides a gain of $26 \%$ in the average user throughput compared to the best SNR association, while the gain in the fifth percentile user throughput reaches $+200 \%$ using a dynamic cell association for min rate maximization.

\section{CONCLUSION}

In this work we have presented a new theoretical framework to study the impact of cell association on the downlink of heterogeneous networks. We have derived upper bounds on the achievable sum rate and min rate and proposed a dynamic range extension algorithm, which outperforms the conventional cell association scheme and comes close to the theoretical limit. In order to validate our results, we have implemented dynamic range extension in an LTE simulator and demonstrated promising performance increase. Future work will consist in considering larger networks with several pairs of macro and pico nodes.

\section{REFERENCES}

[1] NTT-DOCOMO, "Performance of eICIC with Control Channel Coverage Limitation," 3GPP TSG RAN WG1 Meeting 61, R1-103264.

[2] A. Khandekar, N. Bhushan, J. Tingfang, and V. Vanghi, "LTE-Advanced: Heterogeneous networks," in European Wireless Conference, 2010.

[3] S. Das, H. Viswanathan, and G. Rittenhouse, "Dynamic load balancing through coordinated scheduling in packet data systems," in Proc. IEEE INFOCOM 2003, march-april 2003, pp. 786 - 796 vol.1.

[4] A. Sang, X. Wang, M. Madihian, and R. D. Gitlin, "Coordinated load balancing, handoff/cell-site selection, and scheduling in multi-cell packet data systems," Wireless Networks, vol. 14, pp. 103-120, 2006.

[5] T. Bu, L. Li, and R. Ramjee, "Generalized proportional fair scheduling in third generation wireless data networks," in Proc. IEEE INFOCOM 2006, april 2006

[6] K. Son, S. Chong, and G. Veciana, "Dynamic association for load balancing and interference avoidance in multi-cell networks," IEEE Trans. on Wireless Commun., vol. 8, pp. 3566 - 3576, July 2009.

[7] Ericsson and ST-Ericsson, "Aspects on Distributed RRUs with Shared Cell-ID for Heterogeneous Deployments," 3GPP TSG-RAN WG1 Meeting 64, R1-110649.

[8] S. Boyd and L. Vandenberghe, Convex optimization. Cambridge university press, 2008 (sixth printing).

[9] "3GPP TR36.814, Further advancements for E-UTRA physical layer aspects."

[10] "3GPP TR25.996, Spatial channel model for MIMO simulations." 